\title{
Espondiloartropatias: Espondilite Anquilosante e Artrite Psoriásica ${ }^{*}$
}

\author{
Sampaio-Barros PD, Carvalho MAP, Azevedo VF, Campos WR, Carneiro SCS, Giorgi RDN, Gonçalves \\ CR, Hilário MOE, Keiserman MW, Leite NH, Pereira IA, Vieira WP, Vilela EG, Xavier RM, Ximenes AC
}

\section{DESCRIÇÃO DO MÉTODO DE COLETA DE EVIDÊNCIAS}

Reunião consensual para elaboração do texto com inclusão das citações bibliográficas, numa colaboração de reumatologistas com as especialidades de reumatologia pediátrica, dermatologia, gastroenterologia e oftalmologia. Foram convidados 12 reumatologistas responsáveis pelo ambulatório de espondiloartropatias em suas instituições (ou seus representantes); cada participante foi convidado a fazer uma análise crítica, utilizando o conceito da medicina baseada em evidências, de um aspecto diferente dentro do espectro do tratamento das espondiloartropatias. Foi convidado um especialista de cada área para fazer a mesma análise crítica nos tópicos espondiloartropatias juvenis (reumatologista pediátrico), psoríase (dermatologista), doenças inflamatórias intestinais (gastroenterologista) e uveíte anterior (oftalmologista). Após a elaboração de um texto-base de apoio para as discussões, todos os especialistas se reuniram, no período entre 22 e 24 de abril de 2004, a fim de discutir individualmente cada um dos 16 tópicos elaborados e estabelecer um consenso baseado em evidências, a partir do qual os coordenadores redigiram o texto do consenso, submetido à apreciação de todos os participantes para a realização dos ajustes finais.

\section{GRAU DE RECOMENDAÇÃO E FORÇA DE EVIDÊNCIA}

A: Estudos experimentais e observacionais de melhor consistência.

B: Estudos experimentais e observacionais de menor consistência.

C: Relatos de casos, estudos não controlados.

D: Opinião desprovida de avaliação crítica, baseada em consensos, estudos fisiológicos ou modelos animais.

\section{OBJETIVOS}

Oferecer informações sobre a otimização do tratamento das diferentes espondiloartropatias.

\section{CONFLITO DE INTERESSE}

Os autores Sampaio-Barros PD, Carneiro SCS, Hilário MOE, Xavier RM e Ximenes AC declararam vínculo com a Indústria Farmacêutica.

\section{INTRODUÇÃO}

O conceito das espondiloartropatias soronegativas foi estabelecido em 1974, quando os pesquisadores ingleses Moll e Wright propuseram que se englobasse dentro de um mesmo conjunto algumas doenças até então consideradas completamente distintas entre si, mas que na verdade apresentavam diversas características comuns. Estas características englobavam aspectos clínicos (dor axial infla- matória, associada à artrite, predominante em grandes articulações de membros inferiores, e entesopatias periféricas), radiológicos (sacroiliíte) e laboratoriais (soronegatividade para o fator reumatóide, pois, até a década de 70 , alguns pesquisadores consideravam a espondilite anquilosante como o componente axial da artrite reumatóide), em indivíduos com predisposição genética (ligada ao antígeno de histocompatibilidade HLA-B27). Este conjunto incluiu a espondilite anquilosante, a artrite psoriásica, a

* Trabalho realizado por representantes da Sociedade Brasileira de Reumatologia. Elaboração final: 21 de junho de 2004.

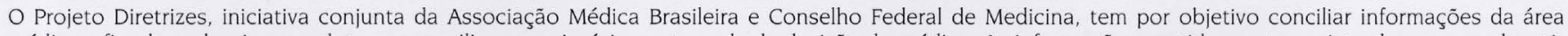

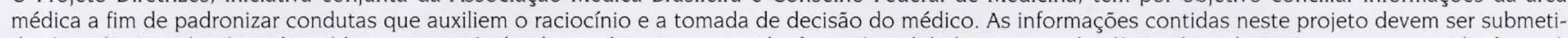

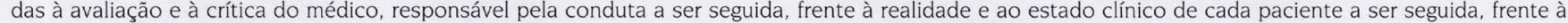
realidade e ao estado clínico de cada paciente. 
artrite reativa e a síndrome de Reiter, e as artropatias enteropáticas (associadas às doenças inflamatórias intestinais $)^{(1)}(\mathbf{C})$. Nos últimos 20 anos, devido ao crescente número de pacientes que apresentam um diagnóstico provável, mas que não conseguem preencher os critérios diagnósticos para uma doença definida dentro do grupo, foi criado o conceito das espondiloartropatias indiferenciadas. Recentemente, especialistas internacionais propuseram a mudança de nome para espondiloartropatias, suprimindo o termo soronegativas ${ }^{(2)}(\mathbf{C})$.

\section{CONSIDERAÇÕES GERAIS SOBRE A ABORDAGEM TERAPÊUTICA DE PACIENTES COM ESPONDILOARTROPATIAS}

As doenças crônicas, como as espondiloartropatias, na maioria das vezes causam um sentimento de desamparo e inutilidade mais assustador do que a doença em si. Por isso, o paciente sempre deve ser informado de forma realista sobre as perspectivas prognósticas de sua doença, perspectivas essas que são freqüentemente otimistas, desde que haja uma abordagem global de atendimento. O paciente deve estar absolutamente ciente de que sua participação especialmente no estar bem consigo mesmo, apesar da doença - será um ponto de partida fundamental para o sucesso terapêutico. O seguimento dos pacientes e o tratamento devem ser realizados de forma individual, caso a caso, já que a história natural da doença pode passar por flutuações durante seu curso evolutivo. O médico deve considerar o impacto da doença para o indivíduo dentro de sua comunidade, com as devidas implicações no seu ambiente familiar e de trabalho, além dos aspectos sociais e financeiros. Apesar de inexistir, até o momento, um tratamento específico para a cura, deve-se ressaltar que os recursos terapêuticos disponíveis possibilitam, de um modo geral, um adequado controle da doença. Um ponto fundamental para tais conquistas está centrado numa boa relação médico-paciente; quando necessário, o médico assistente deve indicar auxílio psicoterápico ou uso de drogas antidepressivas.

\section{ESPONDILITE ANQUILOSANTE}

A espondilite anquilosante é uma doença inflamatória crônica que acomete preferencialmente a coluna vertebral, podendo evoluir com rigidez e limitação funcional progressiva do esqueleto axial. Geralmente se inicia no adulto jovem (segunda a quarta décadas da vida), preferencialmente do sexo masculino, da cor branca e em indivíduos HLA-B27 $\operatorname{positivos}^{(3)}(\mathbf{D})$.

A espondilite de início no adulto, que se inicia a partir dos 16 anos, costuma ter como sintoma inicial a lombalgia de ritmo inflamatório, com rigidez matinal prolongada e predomínio dos sintomas axiais durante sua evolução. A doença juvenil, que se manifesta antes dos 16 anos de idade, costuma iniciar-se com artrite e entesopatias periféricas, evoluindo, somente após alguns anos, para a característica lombalgia de ritmo inflamatório. A doença juvenil costuma ter diagnóstico mais tardio, e muitos destes pacientes podem ser diagnosticados como artrite reumatóide juvenil, no início dos sintomas. O comprometimento do quadril é mais freqüente na criança do que no adulto, o que determina um pior prognóstico, pela necessidade de próteses totais de quadril em muitos pacientes ${ }^{(4)}(B)$.

A freqüente associação com o HLA-B27 faz com que a doença seja mais comum em populações brancas, onde a prevalência do HLA-B27 é significativamente maior. Por sua vez, a positividade do HLA-B27 nos pacientes espondilíticos pode variar entre $80 \%$ a $98 \%$, sendo mais elevada em populações brancas não miscigenadas do norte da $\operatorname{Europa}^{(5)}(\mathbf{D})$. Devido à extrema raridade da presença do HLA-B27 em populações negras africanas, a doença é muito pouco freqüente em negros; no Brasil, país de intensa miscigenação racial, a doença, bem como outras espondiloartropatias, costuma ser encontrada em mulatos (devido à influência da ascendência genética branca), mas é bastante rara em negros não miscigenados $^{(6)}(\mathbf{B})$. Para a confirmação do diagnóstico da doença, os critérios mais utilizados são os de Nova York modificados, que combinam critérios clínicos e radiográficos.

Os critérios clínicos são:

- Dor lombar com mais de três meses de duração que melhora com o exercício e não é aliviada pelo repouso;

- Limitação da coluna lombar nos planos frontal e sagital;

- Expansibilidade torácica diminuída (corrigida para idade e sexo).

Os critérios radiográficos são:

- Sacroiliíte bilateral, grau 2, 3 ou 4;

- Sacroiliíte unilateral, grau 3 ou 4.

Para o diagnóstico de doença, é necessária a presença de um critério clínico e um radiográfico ${ }^{(7)}(\mathbf{C})$.

Inicialmente, o paciente espondilítico costuma queixar-se de dor lombar baixa de ritmo inflamatório, caracterizada por melhorar com o movimento e piorar com o repouso, apresentando rigidez matinal prolongada. A evolução costuma ser ascendente, acometendo progressivamente a coluna dorsal e cervical, contribuindo para o desenvol- 
vimento da "postura do esquiador", caracterizada pela retificação da lordose lombar, acentuação da cifose dorsal e retificação da lordose cervical (com projeção da cabeça para a frente). $\mathrm{O}$ acometimento articular periférico é caracterizado pela presença de oligoartrite e entesopatias. A oligoartrite predomina em grandes articulações de membros inferiores, como tornozelos, joelhos e coxofemorais. Ombros, esternoclaviculares e articulações costocondrais (causando dor torácica) também podem ser acometidos. As entesopatias (inflamações nas inserções dos tendões e/ ou ligamentos nos ossos) costumam ser manifestações iniciais na doença de início juvenil e acometem preferencialmente a inserção de tendão de Aquiles e a fáscia plantar. Quanto às manifestações extra-articulares, a mais freqüente é a uveíte anterior, aguda, unilateral, recorrente; pode ser observada em até $40 \%$ dos pacientes num seguimento prolongado, estando geralmente associada ao HLA-B27 positivo e raramente cursa com seqüielas ${ }^{(8)}(\mathbf{D})$.

Tratamento. Inicialmente, o paciente espondilítico deve ser informado de que, embora a doença seja crônica, apresenta boas perspectivas terapêuticas nos dias de hoje, e de que a agregação familiar da doença somente costuma ser observada em famílias de pacientes HLA-B27 positivo. $\mathrm{O}$ amparo psicológico, visando à perfeita integração à sociedade, é fundamental na condução terapêutica dos pacientes espondilíticos.

A fisioterapia, notadamente os programas de exercícios supervisionados, deve ser realizada de maneira sistemática em todos os estágios da doença, já que os seus benefícios na prevenção de limitações funcionais e na restauração de uma adequada mobilidade articular somente são observados no período em que o paciente realiza os $\operatorname{mesmos}^{(9)}(\mathbf{A})$.

Os antiinflamatórios não hormonais (AINH) devem ser utilizados desde o início do tratamento; não existem trabalhos evidenciando que um determinado AINH se mostre superior aos outros na comparação direta, embora na prática clínica exista o consenso de que a indometacina parece apresentar melhores resultados terapêuticos ${ }^{(10)}(\mathbf{C})$. O uso dos modernos antiinflamatórios Cox-2 específicos, em pacientes com potencial risco de toxicidade gastrointestinal ou que não toleram os AINH convencionais, parece representar uma boa opção para casos de uso prolongado de $\operatorname{AINH}^{(11)}(\mathbf{A})$. Estudo recente demonstra que o uso contínuo dos AINH apresenta melhor resposta quanto aos danos estruturais da doença, em comparação ao uso intermitente dos $\operatorname{mesmos}^{(12)}(\mathbf{D})$.

O uso de corticosteróides é reservado a casos específicos. Em pacientes com artrite periférica persistente, o uso de prednisona, até a dose de $10 \mathrm{mg} /$ dia (ou equivalente) pode ser intermitente, enquanto houver atividade de doenç ${ }^{(13)}(D)$. A utilização de metilprednisolona por via endovenosa não está ainda bem estabelecida, mas pode ser usada em casos muito sintomáticos ${ }^{(14)}(\mathbf{A})$. O uso de corticosteróide por via intra-articular pode ser uma alternativa em casos de artrite persistente ou sacroiliíte refratária (nestes casos, recomendase que a infiltração seja guiada por tomografia computadorizada ou ressonância magnética) ${ }^{(15)}(\mathbf{C})$.

Nos pacientes não responsivos ao uso crônico de AINH devem ser utilizadas drogas de base de ação prolongada. A sulfasalazina, na dose de 30 a $50 \mathrm{mg} / \mathrm{kg} /$ dia, apresenta resposta mais significativa na artrite periférica ${ }^{(16)}(\mathbf{B})$ e na prevenção de surtos recorrentes de uveíte ${ }^{(17)}(\mathbf{A})$. O metotrexato, na dose semanal de 7,5 a $25 \mathrm{mg}$, por via oral ou intramuscular, também apresenta melhor resposta nos pacientes com doença de comprometimento periférico ${ }^{(18)}(\mathbf{B})$, havendo necessidade de melhores estudos para se avaliar sua eficácia nas formas axiais e nas ênteses. Experiências isoladas de especialistas mostram uma tendência a se obter uma boa resposta com o uso da leflunomida ${ }^{(19)}(\mathbf{B})$. O uso de talidomida ${ }^{(20)}(\mathbf{B})$ e de pamidronato $^{(21)}(\mathbf{A})$ tem mostrado resultados iniciais promissores (em estudos isolados e com pequenas casuísticas), mas ainda são necessários estudos controle de qualidade para estabelecer os reais riscos e benefícios de seu uso a médio e longo prazo.

Nos últimos anos, surgiu uma nova classe de drogas de base, os agentes biológicos, que tem demonstrado uma ação bastante eficaz em pacientes refratários ao tratamento convencional. Especificamente, destacam-se o infliximabe e o etanercepte. O infliximabe é uma medicação de uso endovenoso, que requer que sua administração seja realizada em centros de infusão que tenham equipe médica com experiência no acompanhamento destes casos; recomendase que seja administrado na dose de 3 a $5 \mathrm{mg} / \mathrm{kg}$, apresentando um esquema de ataque (doses a 0,2 e 6 semanas) e outro de manutenção, a cada seis a oito semanas, sendo efetivo no controle da atividade da doença ${ }^{(22)}(\mathbf{A})$, na redução dos índices laboratoriais ${ }^{(23)}(\mathbf{B})$, na melhoria da qualidade de $\operatorname{vida}^{(24)}(\mathbf{A})$ e na redução do uso de $\operatorname{AINH}^{(25)}(\mathbf{B})$. O etanercepte é utilizado na dose de $25 \mathrm{mg}$, via subcutânea, duas vezes por semana, sendo efetivo na melhora da dor, função, qualidade de $\operatorname{vida}^{(26)}(\mathbf{A})$ e redução dos índices laboratoriais ${ }^{(27)}(\mathbf{A})$. Na doença juvenil refratária à sulfasalazina e ao metotrexato, os agentes biológicos também podem ser uma boa opção terapêutica ${ }^{(28)}(\mathbf{D})$. É importante se frisar que o uso destas drogas deve obedecer rigorosos critérios de indicação e monitoração, visando diminuir os 
potenciais efeitos colaterais e racionalizar os custos de tratamento ${ }^{(29)}(D)$. Após análise das recomendações para o uso de agentes biológicos nas espondiloartropatias, podemos ratificar os seguintes critérios, propostos pela Canadian Rheumatology Association: o uso do infliximabe e/ou do etanercepte está indicado para a redução de sinais e sintomas de pacientes com espondiloartropatia ativa de moderada a grave intensidade, em indivíduos com resposta inadequada a dois ou mais AINH num período mínimo de observação de três meses, e que não responderam ao uso de metotrexato ou sulfasalazina em casos de artrite periférica em atividade $^{(29)}(\mathbf{D})$. Os estudos atuais mostram ação terapêutica dos agentes biológicos, como monoterapia, por período de até três anos; AINH, metotrexato ou sulfasalazina podem ser usados concomitantemente às drogas anti-TNF, embora não exista nenhuma evidência de uma ação sinérgica entre estas drogas ${ }^{(29)}(\mathbf{D})$. Estudos recentes mostram que o uso crônico do infliximabe por período de dois anos pode impedir a progressão radiológica da doença ${ }^{(30)}(\mathbf{D})$, embora a suspensão da droga após três anos de tratamento possa levar à reagudização da doença ${ }^{(31)}(\mathbf{D})$.

\section{ARTRITE PSORIÁSICA}

A artrite psoriásica é uma artrite inflamatória, soronegativa para o fator reumatóide, associada à psoríase cutânea. A psoríase cutânea é doença bastante freqüente, podendo acometer até $1 \%$ a $2 \%$ da população; em média, $5 \%$ a $10 \%$ dos pacientes com psoríase cutânea evoluirão com artrite psoriásica (este número pode chegar a $40 \%$ em alguns estudos). Costuma iniciar-se entre a terceira e a quinta décadas de vida; a artrite psoriásica juvenil é rara, e em mais da metade dos casos pode manifestar-se inicialmente apenas por artrite (geralmente oligoartrite assimétrica), evoluindo posteriormente para acometimento cutâneo. Não costuma ter predomínio de sexo, exceto em subtipos específicos, com predomínio do sexo feminino na forma poliarticular simétrica e do sexo masculino na forma espondilítica. É mais prevalente em populações brancas. $\mathrm{O}$ acometimento da pele costuma preceder a artrite em 75\% dos casos, havendo início simultâneo em $10 \%$ dos pacientes; nos outros $15 \%$, a artrite pode preceder a lesão de pele. Não é comum haver correlação entre o tipo ou a gravidade da lesão cutânea e a presença, tipo ou extensão do quadro $\operatorname{articular}^{(32)}$ (D).

Embora ainda existam muitas etapas a serem elucidadas na etiopatogenia da artrite psoriásica, acredita-se que, num indivíduo geneticamente predisposto, a presença de um fator ambiental possa funcionar como "gatilho" para desencadear as alterações imunológicas que darão origem à doença. Dentre os fatores ambientais, podemos citar infecção (retrovírus ou bactérias gram-positivas, como o estreptococo, e mais recentemente o HIV) e trauma articular (principalmente em crianças); o estresse emocional representa importante papel no desencadeamento tanto da psoríase cutânea quanto da articular, porém os mecanismos neuro-imuno-endócrinos envolvidos neste processo ainda necessitam ser esclarecidos. Do ponto de vista imunológico, são observadas alterações tanto da imunidade humoral (produção de auto-anticorpos contra antígenos da derme e da membrana sinovial, e presença de imunocomplexos circulantes) quanto da imunidade celular (subpopulações de linfócitos $\mathrm{T}$ ativados na pele e na membrana sinovial) ${ }^{(33)}(\mathbf{D})$.

Classicamente, a artrite psoriásica apresenta cinco formas clínicas:

- Oligoartrite assimétrica (70\%): é a forma clínica mais freqüente, acometendo grandes e/ou pequenas articulações; tenossinovites são comuns, caracterizando os "dedos em salsicha";

- Poliartrite simétrica (15\%): apresenta quadro articular muito semelhante à artrite reumatóide; pode acometer as articulações interfalangeanas distais, comumente não afetadas na artrite reumatóide;

- Distal (5\%): acomete exclusivamente as articulações interfalangeanas distais, geralmente associada a lesões ungueais ("unha em dedal");

- Artrite mutilante $(<5 \%)$ : é a forma clínica menos freqüente e mais grave, acometendo geralmente indivíduos jovens, na segunda e terceira décadas de vida; afeta as pequenas articulações das mãos e dos pés, evoluindo para deformidades importantes, com encurtamento dos dedos;

- Espondilite (5\%): os sintomas clínicos costumam ser indistinguíveis daqueles apresentados pela espondilite anquilosante ${ }^{(34)}(\mathbf{D})$.

$\mathrm{Na}$ última década, chegou-se a propor que a artrite psoriásica fosse dividida em apenas dois subgrupos, o axial e o periférico; no entanto, ainda predomina a classificação em cinco formas clínicas. Alguns autores costumam pleitear a inclusão da Síndrome SAPHO (acrônimo de Sinovite, Acne, Pustulose palmo-plantar, Hiperostose esterno-costoclavicular, Osteíte multifocal crônica recorrente) como um subtipo distinto da artrite psoriásica; no entanto, o assunto está longe de ser um consenso, aliado à extrema raridade da síndrome em nosso país ${ }^{(35)}$ (D).

Tratamento. Dentre as doenças reumáticas auto-imunes, a artrite psoriásica é uma daquelas onde o estresse emocional representa um importante fator desencadeante da crise tanto 
articular quanto cutânea; sendo assim, o suporte psicológico é importante e indispensável na condução dos pacientes psoriásicos, principalmente para assegurar-lhes que a doença é controlável na maioria dos $\operatorname{casos}^{(36)}(\mathbf{D})$. Os AINH são utilizados de rotina no tratamento da artrite psoriásica, cursando com evidente melhora da dor articular, mas não são capazes de alterar sua evolução; devem ser utilizados de forma intermitente, enquanto houver atividade de doença. Não existe nenhum AINH específico comprovadamente melhor que os demais ${ }^{(37)}(\mathbf{D})$; no entanto, é importante salientar que o ácido acetilsalicílico, a indometacina e os oxicams podem exacerbar as lesões da pele ${ }^{(38)}(\mathbf{D})$. Quanto aos corticosteróides, a prednisona em doses baixas (até $10 \mathrm{mg} / \mathrm{dia}$, ou equivalente) pode ser utilizada em casos de artrite periférica persistente, enquanto houver atividade de doença; a retirada da droga deve ser lenta, a fim de se evitar rebote do quadro cutâneo ${ }^{(39)}(\mathbf{D})$; não existem evidências para embasar o uso de altas doses de corticosteróides na artrite psoriásica.

Nos casos não responsivos aos AINH, a droga de primeira escolha é o metotrexato, em doses habituais (por via oral

\section{REFERÊNCIAS}

1. Moll JM, Haslock I, Macrae IF, Wright V. Associations between ankylosing spondylitis, psoriatic arthritis, Reiter's disease, the intestinal arthropathies, and Behcet's syndrome. Medicine 53: 343-64, 1974.

2. Braun J, Sieper J. Building consensus on nomenclature and disease classification for ankylosing spondylitis: results and discussion of a questionnaire prepared for the International Workshop on New Treatment Strategies in Ankylosing Spondylitis, Berlin, Germany, 18-19 January 2002. Ann Rheum Dis 61:61-7, 2002.

3. Van der Linden S, Van der Heijde D. Ankylosing spondylitis: clinical features. Rheum Dis Clin North Am 24:663-76, 1998.

4. Calin A, Elswood J, Rigg S, Skevington SM. Ankylosing spondylitis: an analytical review of 1500 patients: the changing pattern of the disease. J Rheumatol 15:1234-8, 1998.

5. Reveille JD, Ball EJ, Khan MA. HLAB27 and genetic predisposing factors in spondyloarthropathies. Curr Opin Rheumatol 13:265-72, 2001.

6. Sampaio-Barros PD, Bertolo MB, Kraemer MH, Neto JF, Samara AM. Primary ankylosing spondylitis: patterns of disease in a Brazilian population of 147 patients. J R heumatol 28:560-5, 2001.

7. Van der Linden S, Valkenburg HA, Cats A. Evaluation of diagnostic criteria for ankylosing spondylitis: a proposal for modification of the New York criteria. Arthritis Rheum 27:361-8, 1984.

8. Khan MA. Ankylosing spondylitis: clinical aspects. In: Calin A, Taurog JD, editors. Spondylarthritides. Oxford: Oxford University Press; 1998. p.27-40.

9. Dagfinrud H, Hagen K. Physiotherapy interventions for ankylosing spondylitis. Cochrane Database Syst Rev 4:CD002822, 2001. ou intramuscular), até se obter controle da doença40(B). A hepatotoxicidade do metotrexato na artrite psoriásica parece ser maior que a observada em pacientes com artrite reumatóide $^{(41)}$ (D). A sulfasalazina, nas doses de 1 a $3 \mathrm{~g} /$ dia, demonstrou ser eficaz apenas com relação ao componente articular periférico da doença ${ }^{(42)}(\mathbf{A})$. A ciclosporina, nas doses de 3 a $5 \mathrm{mg} / \mathrm{kg} / \mathrm{dia}$, parece ser eficiente tanto para o componente articular quanto cutâneo ${ }^{(43)}(\mathbf{D})$, também apresentando boa resposta quando comparada ao uso do metotrexato $^{(44)}(\mathbf{A})$ e da sulfasalazina ${ }^{(45)}(\mathbf{B})$. Estudo multicêntrico recente enfatiza a boa resposta ao uso da leflunomida, na dose de $20 \mathrm{mg} / \mathrm{dia}^{(46)}(\mathbf{A})$.

De forma semelhante à espondilite, os inibidores do fator de necrose tumoral alfa, infliximabe ${ }^{(47)}(\mathbf{D})$ e etanercepte $^{(48)}(\mathbf{A})$, têm demonstrado resultados satisfatórios tanto na doença articular quanto cutânea, em adultos e crianças. Seu uso deve ser preferencial nos casos refratários aos AINH e drogas de base, como o metotrexato, ciclosporina ou leflunomida. Devem ser obedecidos os mesmos cuidados rigorosos quanto ao seguimento dos pacientes espondilíticos.

10. Calin A, Elswood J. A prospective nationwide cross-sectional study of NSAID usage in 1331 patients with ankylosing spondylitis. J Rheumatol 17:801-3, 1990.

11. Dougados M, Béhier J-M, Jolchine I, et al. Efficacy of celecoxib, a cyclooxygenase 2-specific inhibitor, in the treatment of ankylosing spondylitis: a six-week controlled study with comparison against placebo and against a conventional nonsteroidal antiinflamatory drug. Arthritis Rheum 44:180-5, 2001.

12. Wanders A, Van der Heijde D, Landewé R, et al. Inhibition of radiographic progression in ankylosing spondylitis by continuous use of NSAIDs. Arthritis Rheum 48:S233, 2003.

13. Dougados M, Dijkmans B, Khan M, Maksymowych W, van der Linden S, Brandt J. Conventional treatments for ankylosing spondylitis. Ann Rheum Dis 61:iii40-50, 2002.

14. Peters ND, Ejstrup L. Intravenous methylprednisolone pulse therapy in ankylosing spondylitis. Scand J Rheumatol 21:134-8, 1992.

15. Braun J, Bollow M, Seyrekbasan F, et al. Computed tomography guided corticosteroid injection of sacroiliac joint in patients with spondyloarthropathy with sacroiliitis: clinical outcome and followup by dynamic magnetic resonance imaging. J Rheumatol 23:659-64, 1996.

16. Dougados M, Van der Linden S, Leirisalo-Repo M, et al. Sulfasalazine in the treatment of spondylarthropathy: a randomized multicenter, double-blind, placebocontrolled study. Arthritis Rheum 38:618-27, 1995.

17. Benitez-Del-Castilho JM, Garcia-Sanchez J, Iradier T, Bañares A. Sulfasalazine in the prevention of anterior uveitis associated with ankylosing spondylitis. Eye14:340-3, 2000.

18. Sampaio-Barros PD, Costallat LT, Bertolo MB, Marques Neto JF, Samara AM. Methotrexate in the treatment of ankylosing spondylitis. Scand J Rheumatol 29:160-2, 2000. 
19. Haibel H, Rudwaleit M, Braun J, Sieper J. Leflunomide in ankylosing spondylitis. Ann Rheum Dis 62:FRI0173, 2003.

20. Huang F, Gu J, Zhao W, Zhu J, Zhang J, Yu DT. One-year openlabel trial of thalidomide in ankylosing spondylitis. Arthritis Rheum 47:249-54, 2002.

21. Maksymowych WP, Jhangri GS, Fitzgerald AA, et al. A six-month randomized, controlled, doubleblind, dose-response comparison of intravenous pamidronate $(60 \mathrm{mg}$ versus $10 \mathrm{mg}$ ) in the treatment of nonsteroidal antiinflammatory drug-refractory ankylosing spondylitis. Arthritis Rheum 46:766-73, 2002.

22. Van Den Bosch F, Kruithof, Baeten D, et al. Randomized doubleblind comparison of chimeric monoclonal antibody to tumor necrosis factor alpha (infliximab) versus placebo in active spondylarthropathy. Arthritis Rheum 46:755-65, 2002.

23. Kruithof E, Van den Bosch F, Baeten D, et al. Repeated infusions of infliximab, a chimeric anti-TNF alpha monoclonal antibody, in patients with active spondyloarthropathy: one year follow up. Ann Rheum Dis 61:207-12, 2002.

24. Braun J, Brandt J, Listing J, et al. Treatment of active ankylosing spondylitis with infliximab: a randomized controlled multicentre trial. Lancet 359:1187-93, 2002.

25. Braun J, Brandt J, Listing J, et al. Long-term efficacy and safety of infliximab in the treatment of ankylosing spondylitis: an open, observational, extension study of a threemonth, randomized, placebocontrolled trial. Arthritis Rheum 48:2224-33, 2003.

26. Brandt J, Khariouzov A, Listing J, et al. Sixmonth results of a double-blind, placebocontrolled trial of etanercept treatment in patients with active ankylosing spondylitis. Arthritis Rheum 48:1667-75, 2003.

27. Davis JC Jr, van der Heijde D, Braun J, et al. Recombinant human tumor necrosis factor receptor (etanercept) for treating ankylosing spondylitis: a randomized controlled trial. Arthritis Rheum 48: 3230-6, 2003.

28. Reiff AHM. Prolonged efficacy of etanercept in refractory juvenile ankylosing spondylitis. Arthritis Rheum 44:S292, 2001.

29. Maksymowych W, Inman RD, Gladman D, et al. Canadian Rheumatology Association consensus on the use of anti-tumor necrosis factor alpha directed therapies in the treatment of spondyloarthritis. J Rheumatol 30:1356-63, 2003.

30. Baraliakos X, Wanders A, Rudwaleit $\mathrm{M}$, et al. X-ray progression in patients with ankylosing spondylitis (AS) after 2 years of therapy with infliximab. Ann Rheum Dis 63:412, 2004.

31. Baraliakos X, Brandt J, Listing J, Rudwaleit M, Sieper J, Braun J. Clinical response to withdrawal of anti-TNF therapy in patients with ankylosing spondylitis (AS) after 3 years of continuous treatment with infliximab. Ann Rheum Dis 63:413, 2004.
32. Espinoza LR, Cuellar ML. Psoriatic arthritis and spondylitis: a clinical approach. In: Calin A, Taurog JD, editors. Spondylarthritides. Oxford: Oxford University Press; 1998. p.97-111.

33. Finzi AF, Gibelli E. Psoriatic arthritis. Int J Dermatol 30:1-7, 1991.

34. Gladman DD. Psoriatic arthritis. Rheum Dis Clin North Am 24: 829-44, 1998.

35. Kahn MF, Chamot AM. SAPHO syndrome. Rheum Dis Clin North Am 18:225-46, 1992.

36. Greaves MW, Weinstein GD. Treatment of psoriasis. N Engl J Med 332:581-8, 1995

37. Gladman DD. Effectiveness of psoriatic arthritis therapies. Semin Arthritis Rheum 33:29-37, 2003.

38. Tsankov N, Kazandjieva J, Drenovska K. Drugs in exacerbation and provocation of psoriasis. Clin Dermatol 16:333-51, 1998.

39. Goupille P, Soutif D, Valat J-P. Treatment of psoriatic arthropathy. Semin Arthritis Rheum 21:355-67, 1992.

40. Espinoza LR, Zakraoui L, Espinoza CG, et al. Psoriatic arthritis: clinical response and sideeffects to methotrexate therapy. J R heumatol 19:872-7, 1992.

41. Whiting-O'Keefe QE, Fye KH, Sack KD. Methotrexate and histologic hepatic abnormalities: a meta-analysis. Am J Med 90: 711-6, 1991.

42. Clegg DO, Reda DJ, Mejias E, et al. Comparison of sulfasalazine and placebo in the treatment of psoriatic arthritis. A Department of Veterans Affairs Cooperative Study. Arthritis Rheum 39:2013-20, 1996.

43. Olivieri I, Salvarani C, Cantini F, et al. Therapy with cyclosporine in psoriatic arthritis. Semin Arthritis Rheum 27:36-43, 1997.

44. Spadaro A, Riccieri V, Silli-Scavalli A, Sensi E, Taccari E, Zoppini A. Comparison of cyclosporin A and methotrexate in the treatment of psoriatic arthritis: a one-year prospective study. Clin Exp Rheumatol 13:589-93, 1995.

45. Salvarani C, Macchioni P, Olivieri I, et al. A comparison of cyclosporine, sulfasalazine, and symptomatic therapy in the treatment of psoriatic arthritis. J Rheumatol 28:2274-82, 2001.

46. Kaltwasser JP, Nash P, Gladman D, et al. Efficacy and safety of leflunomide in the treatment of psoriatic arthritis and psoriasis: a multinational, double-blind, randomized, placebo-controlled clinical trial. Arthritis Rheum 50:1939-50, 2004.

47. Antoni CE, Kavanaugh A, Kirkham B, et al. The infliximab multinational psoriatic arthritis controlled trial (IMPACT): substantial efficacy on synovitis and psoriatic lesions with or without concomitant DMARD therapy. Arthritis Rheum 46:S381, 2002.

48. Mease PJ, Goffe BS, Metz J, VanderStoep A, Finck B, Burge DJ. Etanercept in the treatment of psoriatic arthritis and psoriasis: a randomized trial. Lancet 356:385-90, 2000. 